diagnosis; only $20 \%$ had low-risk disease. Of the pretreatment variables examined, only PSA level at diagnosis improved over the duration of the study - the proportion of newly enrolled participants with PSA $<10 \mathrm{ng} / \mathrm{ml}$ increased from $42 \%$ in 2001 to $68 \%$ in 2006 .

The authors suggest that the increased prevalence of high-risk prostate cancer among poor and uninsured men may derive from a lack of access to early detection programs. Although programs like IMPACT extend high-quality cancer treatment to low-income individuals, improved access to screening programs is likely to be necessary to reduce and eliminate disparities in prostate cancer outcomes.

Original article Miller DC et al. (2008) Prostate cancer severity among low income, uninsured men. J Urol 181: 579-584

\section{Sunitinib treatment of unresectable renal cell carcinoma: an initial report}

Agents targeted towards vascular endothelial growth factor might be suitable neoadjuvant treatments for patients with potentially unresectable primary renal tumors. Thomas et al. have now investigated whether one such agent, sunitinib, could induce shrinking of primary renal tumors, potentially allowing surgical tumor resection.

The team treated 19 patients (median age 64 years) with locally advanced and/or metastatic renal cell carcinoma (median tumor size $10.5 \mathrm{~cm}$ ) considered to be unsuitable for resection. Oral sunitinib $(50 \mathrm{mg})$ was administered daily to all patients for 4 weeks, followed by 2 weeks off-treatment. Tumor response assessments were carried out every two cycles.

A complete response was not observed in any of the patients. Primary and overall tumor responses were as follows: partial response in 3 and 2 patients, respectively, stable disease in 7 patients for both, and disease progression in 9 and 10 patients, respectively, with 5 deaths reported in this last group. A decrease in primary tumor size (mean reduction 24\%) was noted in 8 patients, 3 of whom underwent nephrectomy, together with a fourth patient who experienced partial response of metastatic disease to sunitinib without primary tumor shrinkage.

Sunitinib could provide a safe means of treatment for patients with unresectable renal cell carcinoma, reducing tumor burden and facilitating surgical resection. Caution should be taken, however, until further data from larger studies investigating the efficacy of this targeted therapy are available.

Original article Thomas AA et al. (2008) Response of the primary tumor to neoadjuvant sunitinib in patients with advanced renal cell carcinoma. J Urol 181: 518-523

\section{Novel androgen receptor variants in hormone-refractory prostate cancer}

Hu et al. have uncovered a novel mechanism for the development of hormone-refractory prostate cancer. The researchers found seven novel androgen receptor variants that are expressed in prostate cancer cell lines and/or clinical hormone-refractory prostate cancer samples. The variant genes include 'cryptic exons' that encode premature stop codons: the mutant proteins include the $\mathrm{N}$-terminal and DNA-binding domains of the androgen receptor, but lack the ligand-binding domain. The mutations render the variant receptors constitutively active - that is, able to activate androgen-receptor-responsive genes without the requirement for androgen binding.

One of the most abundant variants, designated AR-V7, had 20-fold greater expression in hormone-refractory prostate cancer samples than in hormone-naive specimens, and high AR-V7 expression predicted biochemical recurrence after surgery. Antibodies against AR-V7 detected the protein in 10 of 12 hormonerefractory prostate cancer samples, but in only 1 of 9 hormone-naive samples. Experiments in prostate cancer cell lines showed that, unlike the androgen receptor, AR-V7 localized to the cell nucleus even in the absence of androgen stimulation, and drove the expression of androgen-receptor-responsive genes.

The authors suggest that targeting the androgen receptor $\mathrm{N}$-terminal domain to completely abolish androgen receptor signaling is a possible therapeutic approach for hormonerefractory prostate cancer. In addition, androgen receptor variants might serve as potential biomarkers for advanced prostate cancer.

Original article Hu R et al. (2009) Ligand-independent androgen receptor variants derived from splicing of cryptic exons signify hormone-refractory prostate cancer. Cancer Res 69: $16-22$ 